\title{
Using MACBETH with the Choquet Integral Fundamentals to Model Interdependencies between Elementary Concerns in the Context of Risk Management
}

\author{
Diana F. Lopes ${ }^{1}$, Carlos A. Bana e Costa ${ }^{1}$, Mónica D. Oliveira ${ }^{1}$ and Alec Morton ${ }^{2}$ \\ ${ }^{I}$ CEG-IST, Centre for Management Studies of Instituto Superior Técnico, Universidade de Lisboa, Avenida Rovisco Pais, \\ 1049-001 Lisbon, Portugal \\ ${ }^{2}$ Management Science Department, University of Strathclyde, 16 Richmond Street, Glasgow G1 1XQ, Scotland, U.K. \\ diana.lopes@tecnico.ulisboa.pt,carlosbana@tecnico.ulisboa.pt,monica.oliveira@tecnico.ulisboa.pt, \\ alec.morton@strath.ac.uk
}

Keywords: Interactions, Value Interactions, Choquet Integral, MACBETH, Risk Evaluation.

\begin{abstract}
Effective risk management typically requires the evaluation of multiple consequences of different sources of risk, and multicriteria value models have been used for that purpose. The value of mitigating a risk impact is often considered by risk managers as dependent on the levels of other impacts, therefore there is a need for procedures to identify and model these interactions within a value measurement framework. The Choquet Integral (CI) has been used for this purpose, and several studies in the performance measurement literature have combined the 2-additive CI operator with the MACBETH approach to model interdependencies in real contexts. In this paper, we propose an alternative procedure to model interdependencies and determine the CI parameters from one single MACBETH global matrix. The procedure is illustrated with the construction of a descriptor of impacts to evaluate the risk impacts at ALSTOM Power. The paper further explains the questioning protocol to apply the proposed procedure, as well as how decision-makers can interpret the CI parameters.
\end{abstract}

\section{INTRODUCTION}

Risk management relates to all sources of risk that can affect an organization in terms of its goals and vision (Aven, 2008) (p. 6). Typically, risks are characterized in terms of their probability of occurrence and impact (or consequences) (Williams, 1996) and depending on the context, the risk impact can be evaluated on a single concern or on multiple concerns - also described in the literature as dimensions or attributes (Aven, 2008) (p. 42). For example in a project management context, the concerns may be timely delivery, incurred costs within the budget envelope, and conformance to specifications of the project. Multicriteria value models can assist in evaluating those impacts (Linkov et al., 2006). Value models demand for the structuring of concerns in a value tree, with key concerns (or criteria) being independent axes for the evaluation of impacts. Very often, key concerns are a cluster of interdependent elementary concerns
(EC) (Bana e Costa et al., 1999) (having also been described as interacting criteria (Clivillé et al., 2007; Grabisch et al., 2010)), and therefore modelling should identify and account for possible value interdependencies between EC. This is a major challenge in Multiple Criteria Decision Analysis (MCDA) literature (Grabisch et al., 2010), and several studies have explored the use of Choquet integral (CI) operators in modelling interdependencies. In particular, many of them use an extension of MACBETH (Measuring Attractiveness by a Categorical Based Evaluation Technique) (Bana e Costa et al., 2012) with CI operators (Berrah et al., 2007; Clivillé et al., 2007; Brosig, 2012; Merad et al., 2013). Nonetheless, these applications entail methodological problems.

With the aim of creating a tool to be easily used for decision aid in the context of multicriteria value measurement and to overcome pitfalls from previous studies, this paper exploits an alternative procedure to use MACBETH with the CI, through the use of a 
MACBETH global matrix. The use of the proposed procedure is illustrated with a case of evaluation of interdependent risk impacts at ALSTOM Power.

This paper is organized in four sections. Section 2 presents a literature review. Section 3 proposes the MACBETH global matrix with the CI to model interdependencies, being applied to the context of modelling interdependencies at ASTOM Power. Section 4 presents concluding remarks and describes ongoing research.

\section{LITERATURE REVIEW}

This section starts by clarifying key concepts and the nomenclature in use in this paper. It then reviews previous studies that have modelled interdependencies, emphasizing those that have used $\mathrm{CI}$ operators and MACBETH.

\section{$2.1 \quad$ Nomenclature}

Previous studies modelling interdependencies used a diverse terminology. We start by clarifying the nomenclature adopted in the remaining of this paper. Departing from the value-focused thinking framework of Keeney (1992), we adopt "the approach of structuring concerns" of Bana e Costa et al. (2005), which makes clear the difference between concerns, key concerns (KC) and elementary concerns. A concern (also called "point of view" by Bana e Costa et al. (1999) and "objective" by Keeney (1992) is any aspect within a specific decision context seen as relevant at the eyes of at least one actor for the analysis (Bana e Costa et al., 2005). Stated objectives, points of view, active characteristics of the options and possible consequences of potential options are all concerns (Bana e Costa C. A. et al., 1998). Key concerns are the "ends objectives [concerns]" (Keeney, 1992) that emerge progressively from the structuring phase, and need to respect necessary preferential independence conditions (Bana e Costa et al., 2005), so that they can be isolated from the other KC. Each of them must be measurable and operational by assigning a natural, proxy or indirect, or a constructed descriptor of impacts (i.e., ordered set of plausible impact levels (Bana e Costa et al., 2002; Bana e Costa et al., 2005)). Very often, a KC is defined by a cluster of several interrelated elementary concerns which are usually seen as means to achieve ends (Bana e Costa et al., 1999). It is on the level of the EC that value dependencies (to be modelled with the $\mathrm{CI}$ ) occur.
Since there is no agreement in literature regarding the meaning of concept of interaction or judgmental dependencies, it is useful to clarify this concept. There are two main types of preferential independence conditions: (1) ordinal independence and (2) cardinal independence. The first one is verified when options or alternatives can be ranked with respect to one $\mathrm{KC}$ independent of their impact in other aspects; the second one is verified, when, additionally, the difference in attractiveness between the options can be measured with respect to one $\mathrm{KC}$ independently of their impact in other aspects (Bana e Costa et al., 2005). For instance, consider the following two concerns "peak noise level during the night" and "noise level during the whole day" (Bana e Costa et al., 2005). Whichever the average noise during the day, low peaks during the night are always preferred to high peaks - this is an example of ordinal independence. However, if the difference of attractiveness between two night peak levels depends of the average noise level during the whole day, cardinal dependence occurs (Bana e Costa et al., 2005). In this sense, interactions are related to preferential dependence, and these can be expressed in different ways (Bana e Costa et al., 2005), for instance by antagonism (in which the combined impacts are valued less than the sum of the values of impacts) and by synergism (with the combined impact valuing more than the sum).

Marichal (2000) suggests the existence of three types of interactions: (1) correlation, (2) complementarity/substitutiveness and (3) preferential dependence. In the scope of our study we suggest that correlations should not be considered because they refer to environmental (physical or statistical) and not judgmental relations between concerns (von Winterfeldt D. et al., 1986). E.g., the costs and safety of a road infrastructure are statically correlated, in the sense that higher safety usually requires higher investments, but they can be taken as two (judgmentally independent) key concerns, because the DM is able to rank levels from one $\mathrm{KC}$ and can judge the difference in attractiveness between any two impact levels from one $\mathrm{KC}$ without knowing their impact on the other $\mathrm{KC}$. Hence, in contrast to what it is stated in other studies such as Shah et al. (2013) and Marichal (2000), correlations can be ignored unless they are redundant (Bana e Costa et al., 2005). On the other hand, we believe that substitutiveness and complementarity are types of preferential dependence, more precisely extreme situations of antagonism and synergism, respectively. For example, substitutiveness corresponds to a situation 
when the combined impact is valued less than the sum of individual impacts, being the value of the individual impacts close to the value of the combined impact (Grabisch et al., 2004). Hence, there is no need to distinguish between preferential or value dependence and substitutiveness/ complementarity.

\subsection{MACBETH basics}

MACBETH is a multicriteria decision analysis approach that requires only non-numerical judgments about differences in attractiveness between options to help the decision maker (DM) measure the relative attractiveness or value of options (Bana e Costa et al., 2012). Within a multicriteria value measurement framework, MACBETH has been most commonly used to build value functions and weight criteria in additive models. For building value functions, the MACBETH questioning protocol consists in asking the DM to qualitatively judge the difference in attractiveness between impact levels, two at a time, based on seven semantic categories: "is there no difference, or is the difference very weak, weak, moderate, strong, very strong, or extreme?" (Bana e Costa et al., 2012). During this questioning protocol, a matrix with the categorical judgments of the DM is populated. Each time a qualitative judgment is introduced in the matrix, the M-MACBETH DSS verifies its consistency and offers suggestions to solve eventual inconsistencies (Bana e Costa et al., 2008). After the consistency verification, the software derives, by mathematical programming, an interval numerical scale which has to be analysed and validated by the DM (Bana e Costa et al., 2012). A slightly different procedure than the one just described has been used for weighting EC with CI and MACBETH. Previous studies have shown that MACBETH provides a simple and transparent approach in modelling complex multidimensional problems, and hence its wide applicability in MCDA (examples are (Bana e Costa et al., 1999; Bana e Costa et al., 2002; Carnero, 2006; Bana e Costa et al., 2008; Barin et al., 2012)).

As described above, in its most common use, MACBETH applications use an additive value model by taking "difference independence" as a modelling hypothesis (Bana e Costa et al., 2012). As mentioned in Edwards et al. (1994) (p. 315) "violations of conditional monotonicity, usually easy to detect judgmentally, mean that additive models should not be used", and interdependent EC should be combined/aggregated to form a KC (Bana e Costa et al., 2005).

\subsection{Modelling interdependent EC}

For cases of interdependent EC, there has been an increase in studies using procedures with operators of CI family that belong to the non-additive measure family. These CI operators are represented by a mathematical expression that aggregates impact values of multiple interdependent EC into a single global score (Mayag et al., 2011).

Several studies have applied these CI operators in many evaluation contexts such as to: (i) evaluate supplier's performance (Clivillé et al., 2006; Berrah et al., 2007; Feyziog̃lu et al., 2010), employees' performance (Gürbüz, 2010) and action plans in an organization in industrial contexts (Clivillé et al., 2007; Merad et al., 2013); (ii) assess academic performance of faculty members in the education sector (Cardin et al., 2013); (iii) evaluate classical swine fever control strategies in the animal health literature (Brosig, 2012), (iv) evaluate a new waste incinerator plant location in the environment context (Bottero et al., 2013); and (iv) more recently, in the risk management context, to build a value- and riskbased performance measurement and management system (Vernadat et al., 2013).

Some of these studies lack information about the questioning protocol applied and do not explain how to convert impacts into value or how to determine the CI parameters (Feyziog̃lu et al., 2010). Other studies convert quantitative impacts into values linearly and therefore presuppose that a unit of impact has always the same value, which is a strong assumption (in other words, problems on the interpretation of commensurate scales - see section 3.1) (Bottero et al., 2013; Cardin et al., 2013). Other studies weight EC based only on the notion of importance (Labreuche C. et al., 2005; Brosig, 2012) with no reference to impact ranges - in fact, this has been described as the most common critical mistake in decision analysis (Keeney, 1992). Some studies require a direct elicitation of numerical information about the overall value of each option from the DM (Grabisch M. et al., 2000; Grabisch M. et al., 2008; Merad et al., 2013), there being many practical and theoretical issues regarding this type of elicitation, as discussed by Morton et al. (2009). Several studies make use of local judgments, instead of global judgments, to model interdependencies between EC - examples are (Clivillé et al., 2006; Clivillé et al., 2007; Gürbüz, 2010; Merad et al., 2013; Vernadat et al., 2013), with most of these studies using the CI 
with MACBETH. By local judgment we mean a judgment involving one EC alone; by global judgment we mean a judgment involving the interrelated EC all together. Nonetheless, the use of local judgments is incoherent because under the presence of interdependencies between several EC, one cannot assess local judgments in an EC without considering the levels of impact in the remaining EC. To illustrate this problem, we recall the study by Clivillé et al. (2007) that evaluates four actions to improve the profit margins of a company using CI with MACBETH. One KC concern of this study is "service rate", composed by three EC: "average delay", "order receive time" and "accuracy shipments". Regarding local judgments for the "average delay" EC, the DM evaluated as "extreme" ("6", see the highlighted judgment in Fig. 1(a)) the difference in attractiveness between an action "TI" and a "neutral" action $(\mathrm{N})$. However, if there exist interdependencies between EC, that judgment may change with the impacts in the remaining EC and therefore these should be fixed before the questioning (a further explanation on this is provided in sections 2.4 and 3.3). In addition to the previous problem, some studies such as Clivillé et al. (2006), Clivillé et al. (2007) and Gürbüz (2010) make use of two types of matrices - a first matrix with local judgments (see Fig. 1(a)) and a second with global judgments (see Fig. 1(b)) - that can be problematic. Even if the local judgments, present in the first matrix, were asked to the DM in the form of global judgments, the use of two matrices could entail incoherence and inconsistency problems. To expose these problems, we recall once again the study by Clivillé et al. (2007). If the "good" and "neutral" actions present in Fig. 1(a) were taken as being "neutral" in the remaining EC, i.e. globally comparing the difference in attractiveness between two actions $\left(\mathrm{G}_{1} \mathrm{~N}_{2} \mathrm{~N}_{3}\right)$ and $\left(\mathrm{N}_{1} \mathrm{~N}_{2} \mathrm{~N}_{3}\right)$, the same global judgment is also present in Fig. 1(b) - see the highlighted judgment ("weak"). However, that judgment is inconsistent with the highlighted judgment in Fig. 1(a). In fact, the difference in attractiveness between an action "TI" (which is less attractive than a "good" action in terms of "average delay") and a "neutral" action (N) is "extreme", which implies that the difference in attractiveness between a "good" and a "neutral" action needs to be "extreme", being inconsistent with the previous "weak" judgment. Taking into account the limitation associated to the use of separated matrices, this paper exploits the use of global judgments within one single MACBETH global matrix. In addition, it is worthwhile to note that some studies such as
Clivillé et al. (2006), Clivillé et al. (2007) and Vernadat et al. (2013) apply MACBETH to build value functions based just on the set of local judgments between consecutive ordered levels given by the DM (diagonal of the MACBETH matrix Fig. 1(a)). However, according to Bana e Costa et al. (2008), "it is recommended to ask for some additional judgments to perform a number of consistency checks". If MACBETH is only applied to the diagonal of the matrix, the procedure does not make use of all the potential of the MACBETH method and DSS. Thus, no consistency checks are made and the MACBETH judgments are used to solve an equation system by attributing a single number to each semantic category (with 'very weak' $=1$, 'weak' $=2, \ldots$, and 'extreme' $=6$ ) - see Fig. 1 - which is an arbitrary assumption. Therefore, it seems also important to overcome these issues, as other numerical values or ranges can be attributed to each semantic category, and consistency checks are also a key feature for a procedure to be used in real decision aid settings.

This study exploits the use of MACBETH with the $\mathrm{CI}$ to model interdependencies and overcoming the limitations pointed above, using a single MACBETH matrix with global judgments and all the features of the MACBETH method and DSS. The aim is to propose a tool that can easily be used for decision support, as it will be described in section 3 .

\subsection{Choquet integral operators}

This section introduces basic concepts regarding CI operators, and a brief explanation of the meaning of CI parameters. The operators of the CI family, introduced by Choquet (1953), belong to the nonadditive measures family and are being applied to model interdependencies between EC. Since we are dealing with interdependencies, the basic idea behind these operators consists in questioning the DM about their preferences on a set of "binary options" to determine the value of the CI parameters - Shapley and interaction parameters, which we describe below. As suggested by the name, these binary options must perform with superior and inferior reference levels on each EC. Depending on the context, best and worst, or good and neutral references, or other superior and inferior references can be used (for instance, the good corresponds to an undoubtedly attractive level and neutral to a level which is neither attractive nor repulsive 


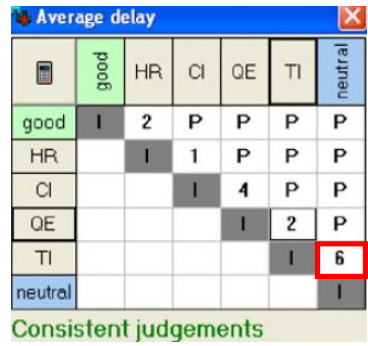

(a)

\begin{tabular}{|c|c|c|c|c|c|c|c|}
\hline & $(\mathbf{N}, \mathbf{G}, \mathbf{G})$ & $(\mathbf{G}, \mathbf{N}, \mathbf{G})$ & $(\mathbf{G}, \mathrm{G}, \mathrm{N})$ & $(\mathbf{N}, \mathbf{N}, \mathbf{G})$ & $(\mathbf{N}, \mathbf{G}, \mathbf{N})$ & $(\mathrm{G}, \mathrm{N}, \mathrm{N})$ & $(\mathrm{N}, \mathrm{N}, \mathrm{N})$ \\
\hline$(\mathbf{N}, \mathbf{G}, \mathbf{G})$ & Null & Moderate (3) & & & & & \\
\hline$(\mathbf{G}, \mathbf{N}, \mathbf{G})$ & & Null & Extreme 6 & & & & \\
\hline$(\mathrm{G}, \mathrm{G}, \mathrm{N})$ & & & Null & Null (0) & & & \\
\hline$(\mathbf{N}, \mathbf{N}, \mathbf{G})$ & & & & Null & Weak (2 & & \\
\hline$(\mathrm{N}, \mathrm{G}, \mathrm{N})$ & & & & & Null & V. Weak (1) & \\
\hline$(\mathbf{G}, \mathbf{N}, \mathbf{N})$ & & & & & & Null & Weak (2) \\
\hline$(\mathrm{N}, \mathrm{N}, \mathrm{N})$ & & & & & & & Null \\
\hline
\end{tabular}

(b)

Figure 1: (a) Local judgments used to build the value function on the EC "average delay" and (b) the matrix that compares interdependent EC (global judgments). Note that HR, CI, QE and TI are abbreviations of the actions that are being evaluated, 'human resources', 'continuous improvement', 'quality and environment' and 'technical investment', respectively (Clivillé

2007).

(Mayag et al., 2011) at the eyes of the DM; and the best corresponds to the most attractive level and the worst to the most repulsive level (Grabisch et al., 2004)). Using the best and worst references, options which are best and/or worst on all the $m$ EC are used in the $\mathrm{CI}$ - for instance $\left(\mathrm{B}_{1} \mathrm{~B}_{2} \mathrm{~W}_{3} \ldots \mathrm{W}_{\mathrm{m}}\right)$, is a binary option which is in the best (B) level on EC 1 and 2 and in the worst $(\mathrm{W})$ level on the remainder $\mathrm{EC}$, in a context of $m$ EC.

To better understand the reason why we should ask the DM about their preferences on the set of binary options, consider a case with three EC and two swings: (i) a swing from an option $\left(\mathrm{W}_{1} \mathrm{~W}_{2} \mathrm{~W}_{3}\right)$ to an option $\left(\mathrm{B}_{1} \mathrm{~W}_{2} \mathrm{~W}_{3}\right)$ and (ii) another swing from $\left(\mathrm{W}_{1} \mathrm{~B}_{2} \mathrm{~W}_{3}\right)$ to $\left(\mathrm{B}_{1} \mathrm{~B}_{2} \mathrm{~W}_{3}\right)$. These swings just differ on the level of EC 2 - worst and best in the first and second swings, respectively. In the context of independent EC, both swings are equally valued, and there is no need to take into account of the different baselines. Under the presence of value interdependencies, these swings are differently judged and one needs to consider all the swings with different baselines.

To apply these operators, the monotonicity condition is required. E.g., consider the following sets of elementary concerns $\mathrm{S}, \mathrm{T}$ and $\mathrm{M}$, and their corresponding complement sets $\mathrm{S}^{\mathrm{c}}, \mathrm{T}^{\mathrm{c}}$ and $\mathrm{M}^{\mathrm{c}}$; if $\mathrm{S} \subseteq$ $\mathrm{T} \subseteq \mathrm{M}$, the option which is at the best level in all the $\mathrm{EC}$ present in $\mathrm{T}$ and at the worst level in the $\mathrm{EC}$ present in $\mathrm{T}^{\mathrm{c}}\left(\mathrm{B}_{\mathrm{T}}, \mathrm{W}_{\mathrm{T}}{ }^{\mathrm{c}}\right)$ dominates the option $\left(\mathrm{B}_{\mathrm{S}}, \mathrm{W}_{\mathrm{S}}{ }^{\mathrm{c}}\right)$ which is in the best level in all the EC from $\mathrm{S}$ and in the worst level in the EC from $\mathrm{S}^{\mathrm{c}}$, because the former is at least as attractive as the latter, then:

$$
\mathrm{S} \subseteq \mathrm{T} \subseteq \mathrm{M} \Rightarrow \mathrm{V}_{\mathrm{Ag}}\left(\mathrm{B}_{\mathrm{T}}, \mathrm{W}_{\mathrm{T}^{\mathrm{c}}}\right) \geq \mathrm{V}_{\mathrm{Ag}}\left(\mathrm{B}_{\mathrm{S}}, \mathrm{W}_{\mathrm{S}^{\mathrm{c}}}\right)
$$

This condition illustrates that the EC cannot be modelled as ordinally dependent. After having information from the DM regarding the numerical values of the binary options, the CI is applied, making use of the following formula - eq. system (2), the general formulation of CI operators.

$$
\left\{\begin{array}{c}
\mathrm{v}_{\mathrm{Ag}}(\mathrm{u})=\sum_{\mathrm{i}=1}^{\mathrm{m}}\left[\left(\mathrm{v}_{(\mathrm{i})}\left(\mathrm{x}_{(\mathrm{i}) \mathrm{u}}\right)-\mathrm{v}_{(\mathrm{i}-1)}\left(\mathrm{x}_{(\mathrm{i}-1) \mathrm{u}}\right)\right) \times\right. \\
\left.\mathrm{V}_{\mathrm{Ag}}\left(\left\{\left(\mathrm{B}_{(\mathrm{i})} \mathrm{B}_{(\mathrm{i}+1)} \ldots \mathrm{B}_{(\mathrm{m})}\right)\right\}\right)\right] \\
\mathrm{v}_{(0)}\left(\mathrm{x}_{(0) \mathrm{u}}\right)=0 \\
\mathrm{v}_{(1)}\left(\mathrm{x}_{(1) \mathrm{u}}\right) \leq \mathrm{v}_{(2)}\left(\mathrm{x}_{(2) \mathrm{u}}\right) \leq \ldots . \mathrm{v}_{(\mathrm{m})}\left(\mathrm{x}_{(\mathrm{m}) \mathrm{u}}\right)
\end{array}\right.
$$

Where $\mathrm{V}_{\mathrm{Ag}}$ represents the aggregated value (global score) of an option $\mathrm{u}, \mathrm{v}_{(\mathrm{i})}$ corresponds to the $i$-th overall value of the option $u$ in ascending order thatis related to a specific EC and baseline (for instance, all worst or all best) in the other EC, $\mathrm{x}_{(\mathrm{i}) \mathrm{u}}$ represents the impact level of the option $u$ in the EC $\mathrm{i}$ and $\mathrm{V}_{\mathrm{Ag}}\left(\left\{\mathrm{B}_{(\mathrm{i})}\right\}\right)$ the aggregated value of an option which is in the best level in the EC $\mathrm{i}$ and in the worst level in the other EC. Note that this mathematical formulation is coherent with the impact values $\left(\mathrm{V}_{\mathrm{Ag}}\right.$ and $\mathrm{v}_{(\mathrm{i})}$ ) on an unipolar scale i.e. the impact values are in the $[0,1]$ range (Grabisch, 2005).

The exponential complexity of the general formula of the CI has shown to be prohibitive in many applications (Grabisch, 2005; Grabisch et al., $2010)$, as $2^{\mathrm{m}}-2$ variables need to be determined, corresponding to the aggregated value of all the binary options $\left(2^{\mathrm{m}}\right)$ excluding the known aggregated values of the options which are worst all over and best all over, that for convention, value 0 and 1 , respectively (Grabisch et al., 2010). To overcome this issue, particular CI operators have been proposed, such as the k-additive ones, highly spread in decision analysis literature. K-additivity fixes the degree of interaction between EC: 1-additivity does 
not allow interaction between EC (corresponding to an additive model); 2- additivity allows interaction up to $2 \mathrm{EC}$, etc. Note that a k-additive operator, that allows interaction up to $\mathrm{k} \mathrm{EC}$, requires the definition of $\sum_{\mathrm{a}=1}^{\mathrm{k}}\left(\begin{array}{c}\mathrm{m} \\ \mathrm{a}\end{array}\right)$ parameters, which makes, in practice, the 2-additivity the best compromise between complexity and richness of the model (Grabisch et al., 2010). In the 2-additivity case the mathematical formulation is given by a linear part, identical to the additive model, modified by effects coming from the interaction represented by the second parcel (see the first equation of the eq. system (3)). Grabisch (1997) explains how to obtain the eq. system (3) from (2).

$$
\left\{\begin{array}{c}
V_{\text {Ag }}(u)=\sum_{i=1}^{m} s_{i} v_{i}\left(x_{i u}\right)-\frac{1}{2} \sum_{\substack{\{i, j\}\} \\
i \neq j}} I_{i j}\left|v_{i}\left(x_{i u}\right)-v_{j}\left(x_{j u}\right)\right| \\
\sum_{i=1}^{m} s_{i}=1
\end{array}\right.
$$

where $v_{i}$ corresponds to the overall value of the option $u$ on the EC $i$ considering a specific baseline (for instance, all worst or all best) in the other EC; $\mathrm{s}_{\mathrm{i}}$ corresponds to the Shapley parameter of the EC $\mathrm{i}$ (see section 3.3); $\mathrm{I}_{\mathrm{ij}}$ the interaction parameter between the EC $\mathrm{i}$ and $\mathrm{j}$.

The Shapley and the interactions parameters are key concepts for the understanding and analysis of interdependencies between EC. As remarked in Grabisch (1997) (p. 171), the Shapley value "can be interpreted as a kind of average value of the contribution of element $\mathrm{i}$ [EC $\mathrm{i}$ in our study] alone in all coalitions [considering different baselines in the other EC]'. With regard to the interaction parameter between 2 EC for instance, it "can be interpreted as a kind of average value of the added value given by putting $\mathrm{i}$ and $\mathrm{j}$ together, all coalitions being considered" (Grabisch, 1997) (p. 171), i.e. an average of the added value given by putting together the 2 EC together, being all the baselines considered. The interaction parameters $\mathrm{I}_{\mathrm{ij}}$ range in the interval [1,1] with: (a) $\mathrm{I}_{\mathrm{ij}}>0$ for a synergistic behaviour between EC i and j, (b) $\mathrm{I}_{\mathrm{ij}}<0$ for an antagonistic behaviour between EC $i$ and $j$, and (c) $I_{i j}=0$ when there is no interaction between EC $\mathrm{i}$ and $\mathrm{j}$. In the next section, the mathematical formulations - see Eqs. (4) and (7) - of these CI parameters are represented and examples are used to illustrate these definitions to DM.

\section{METHODOLOGICAL FRAMEWORK}

Departing from a real case study in risk management, this section explains how the use of a global MACBETH matrix with the CI can be applicable to properly model interdependencies between EC. Special emphasis is also given to the protocol of questioning and to providing an intuitive interpretation of the parameters of the CI that can be explained to a DM.

\subsection{Structuring the evaluation problem}

A key business area of ALSTOM Power is to construct and commission power plants. When building power plants, ALSTOM needs to prioritize risks that can threaten the construction projects. In a real case study (Figueiredo et al., 2009) developed with a group of ALSTOM risk managers (the DM), one KC identified was" Health and Safety" which refers to injuries resulting from incidents that occur inside site barriers, and takes into account two interdependent EC: (a) number of injuries and (b) severity of the injury.

For applying the CI, in each EC a descriptor of impacts and two reference levels within the descriptor need to be defined. The use of reference levels in every EC ensures commensurateness between EC (Grabisch et al., 2004). "Commensurateness means that one shall be able to compare any element of one point of view [EC in our study] with any element of any other point of view" (Grabisch et al., 2004) (p. 565), by considering a baseline defined by those reference levels since we are in the presence of interdependencies. As described in section 2.4, depending on the evaluation context, the reference levels can be defined as best and worst (Labreuche et al., 2003) or as good (G) and neutral (N) (Mayag et al., 2011) levels or as other superior and inferior references. It is important to note that in addition to the two reference levels - which in the ALSTOM example, are the "best" and the "worst" levels other impact levels can be defined. This is the case of descriptors in tables 1 and 2.

Table 1: Descriptor of impacts for the EC 1 "Number of injuries".

\begin{tabular}{|c|c|}
\hline Levels of impact & Description \\
\hline $\mathrm{B}=$ Best & 0 \\
\hline $\mathrm{I}$ & 3 \\
\hline $\mathrm{W}=$ Worst & 10 \\
\hline
\end{tabular}


Table 2: Descriptor of impacts for the EC 2 "Severity of the injury".

\begin{tabular}{|c|c|}
\hline Levels of impact & Description \\
\hline $\mathrm{B}=$ Best & First aid incident (FAI) \\
\hline $\mathrm{M}$ & Medical treatment incident (MTI) \\
\hline $\mathrm{L}$ & Loss time incident (LTI) \\
\hline $\mathrm{W}=$ Worst & Fatality (Fatal.) \\
\hline
\end{tabular}

Since we are dealing with interdependencies and global preference modelling, the next step is to define all the combinations of different impact levels across EC, from tables 1 and 2), being then pairwise compared in a global MACBETH matrix (see the next section). In our example, 12 combinations of impacts are considered (from crossing impact levels of tables 1 and 2, leading to table 3 ). Note in table 3 that if there are no injuries, the impact level of the EC 2 is irrelevant, and so all levels in the constructed descriptor which have no injuries as a component are evaluated as being indifferent.

Table 3: Constructed descriptor of impacts for the $\mathrm{KC}$ "Health and Safety" (B and W stand for Best and Worst, respectively; and the indices refer to the EC 1 and EC 2).

\begin{tabular}{|c|c|}
\hline Levels of impact & Description \\
\hline $\mathrm{B}_{1} \mathrm{~B}_{2} ; \mathrm{B}_{1} \mathrm{M}_{2} ; \mathrm{B}_{1} \mathrm{~L}_{2} ; \mathrm{B}_{1} \mathrm{~W}_{2}$ & No injury \\
\hline $\mathrm{I}_{1} \mathrm{~B}_{2}$ & $3 \mathrm{FAI}$ \\
\hline $\mathrm{W}_{1} \mathrm{~B}_{2}$ & $10 \mathrm{FAI}$ \\
\hline $\mathrm{I}_{1} \mathrm{M}_{2}$ & $3 \mathrm{MTI}$ \\
\hline $\mathrm{W}_{1} \mathrm{M}_{2}$ & $10 \mathrm{MTI}$ \\
\hline $\mathrm{I}_{1} \mathrm{~L}_{2}$ & $3 \mathrm{LTI}$ \\
\hline $\mathrm{W}_{1} \mathrm{~L}_{2}$ & $10 \mathrm{LTI}$ \\
\hline $\mathrm{I}_{1} \mathrm{~W}_{2}$ & 3 fatalities \\
\hline $\mathrm{W}_{1} \mathrm{~W}_{2}$ & 10 fatalities \\
\hline
\end{tabular}

\subsection{Value measurement with MACBETH}

Many studies, such as Berrah et al. (2007) and Clivillé et al. (2007), use MACBETH in a first step to build, what these authors name "elementary expressions" (value functions) in each EC by using local judgments (as shown in Fig. 1(a)); and in a second step to construct a matrix to compare pairs of EC (a matrix requiring global judgments - as shown in Fig. 1(b)). As described in detail in section 2.3, only a simplified version of MACBETH is used in these studies, and the judgments used in building 'value functions' cannot be local judgments; and if they are global judgments, they coincide with some of the judgments used in the second step of 'building weights' (see Fig. 1(b)), which demand for consistency checks when filling the matrices. The use of a single MACBETH matrix of global judgments can avoid these judgmental inconsistencies. Also, using such a matrix allows for accounting for DM's differences of opinion or hesitation that are relevant in real decision contexts.

Accordingly, we propose using one global MACBETH matrix with all the judgments comparing all the levels of the constructed descriptor from Table 3. The rationale behind the use of a global matrix is as follows:

- under the presence of interdependencies, all the judgments required to evaluate impact levels are global;

- the use of a single global matrix allows for depicting cases of inconsistencies between judgments, as well as allows for a clear and simple protocol of questioning DM; also cases of differences of opinion or hesitation can populate the matrix;

- $\quad$ and the numerical scales generated by applying the MACBETH approach to the global matrix can be used not only with the CI, but also to test whether other non-additive models are appropriate (we do not pursue this research line in this study).

Thus, we propose using a single global matrix, such as the one depicted in Fig. 2(a) (making use of all the levels of impact of Table 3). MACBETH is then used with the DM to quantify the relative attractiveness of the impact levels through a qualitative pairwise comparison questioning mode similar to the one described in section 2.2. For instance, reading Fig. 2(a), the DM judged as "moderate" the difference of attractiveness between "no injury" and "3 FAI". It should be emphasized that this is a global judgment for it involves the impact levels in the two EC, according to the constructed descriptors described in Table 3.

Fig. 2 presents relevant information from the single global MACBETH matrix in the $M$ MACBETH DSS: Fig. 2(a) presents the matrix of judgments filled with the DM. Note that disagreement or hesitation between two or more semantic categories when making judgments is allowed - e.g. 'mod-strg', 'strg-vstrg' and 'vstrg'extr' in Fig. 2(a), corresponding to 'moderate or strong', 'strong or very strong' and 'very strong or extreme', respectively. As this matrix is consistent, M-MACBETH DSS proposes a numerical scale that can be adjusted until an interval scale validated by the DM is achieved (Fig. 2(b)). Note that in Fig. 2(c) the semantic categories are quantified by more than one single number. 
Judgments in Fig. 2(a) show an example of judgmental dependencies between the two EC. For instance, the DM judged the difference in attractiveness between no FAI $\left(\mathrm{B}_{1} \mathrm{~B}_{2}\right)$ and $10 \mathrm{FAI}$ $\left(\mathrm{W}_{1} \mathrm{~B}_{2}\right)$ to be 'moderate or strong'; and the difference between no fatality $\left(\mathrm{B}_{1} \mathrm{~W}_{2}\right)$ and 10 fatalities $\left(\mathrm{W}_{1} \mathrm{~W}_{2}\right)$ as 'extreme'- see the highlighted judgments in Fig. 2(a). These judgments indicate that the EC "number of injuries" is cardinally value dependent with the EC "severity of the injury"; and other judgments also suggest that the EC "severity of the injury" is cardinally value dependent with the EC "number of injuries".

Once determined the value scores of all the combinations (see Fig. 2(b)), the next step consists in calculating and intuitively interpreting the CI parameters.

\subsection{Interpretation of the CI parameters}

The Shapley parameters can be interpreted as an average value of the contribution of EC $\mathrm{i}$ considering different baselines in the other EC (all worst, all best, all the combinations with worst and best levels). Its mathematical representation is given by eq. system (4). In the context of independency, the Shapley parameters act like 'weights' in the additive model (Grabisch et al., 2010), in the sense that the contribution of an EC $\mathrm{i}$ is the same, independently of the chosen baseline to the remainder EC.

$$
\left\{\begin{array}{l}
\mathrm{s}_{\mathrm{i}}=\sum_{\mathrm{K} \subset \mathrm{N} \backslash \mathrm{i}} \frac{(\mathrm{m}-|\mathrm{K}|-1) !|\mathrm{K}| !}{\mathrm{n} !}\left[\mathrm{V}_{\mathrm{Ag}}\left(\mathrm{B}_{\mathrm{K}} \cup \mathrm{B}_{\mathrm{i}}\right)-\mathrm{V}_{\mathrm{Ag}}\left(\mathrm{B}_{\mathrm{K}}\right)\right] \\
\sum_{\mathrm{i}=1}^{\mathrm{m}} \mathrm{s}_{\mathrm{i}}=1
\end{array}\right.
$$

To better explain this parameter to a DM, consider the EC "number of injuries" of our case study. The Shapley parameter for this EC is given by an average of the value of two swings, e.g., averaging the value from improving from 10 fatalities $\left(\mathrm{W}_{1} \mathrm{~W}_{2}\right)$ to no fatality $\left(\mathrm{B}_{1} \mathrm{~W}_{2}\right)$ with the value from improving from $10 \mathrm{FAI}\left(\mathrm{W}_{1} \mathrm{~B}_{2}\right)$ to no FAI $\left(B_{1} B_{2}\right)$, the two cases depicted in Fig. 3. Note that both swings depict the case from going to the worst to the best level in the first EC, and when different levels of the second EC are fixed. This means that with interdependent EC the same swing needs to be evaluated together with different baselines. Using the rescaled value scores (from Fig. 2(b)) in eq. system (4), the Shapley parameter for "number of injuries" is:

$\left\{\begin{array}{l}\mathrm{s}_{1}=\frac{1}{2} \times\left[\mathrm{V}_{\mathrm{Ag}}\left(\mathrm{B}_{1} \mathrm{~W}_{2}\right)-\mathrm{V}_{\mathrm{Ag}}\left(\mathrm{W}_{1} \mathrm{~W}_{2}\right)\right]+\frac{1}{2} \times\left[\mathrm{V}_{\mathrm{Ag}}\left(\mathrm{B}_{1} \mathrm{~B}_{2}\right)-\mathrm{V}_{\mathrm{Ag}}\left(\mathrm{W}_{1} \mathrm{~B}_{2}\right)\right] \\ =\frac{1}{2} \times\left(\frac{45}{45}-\frac{0}{45}\right)+\frac{1}{2} \times\left(\frac{45}{45}-\frac{39}{45}\right)=\frac{17}{30}\end{array}\right.$

The same logic is applied to calculate the Shapley parameter for the EC "severity of the injury". I.e., averaging the value of two swings: going from 10 fatalities $\left(\mathrm{W}_{1} \mathrm{~W}_{2}\right)$ to $10 \mathrm{FAI}\left(\mathrm{W}_{1} \mathrm{~B}_{2}\right)$

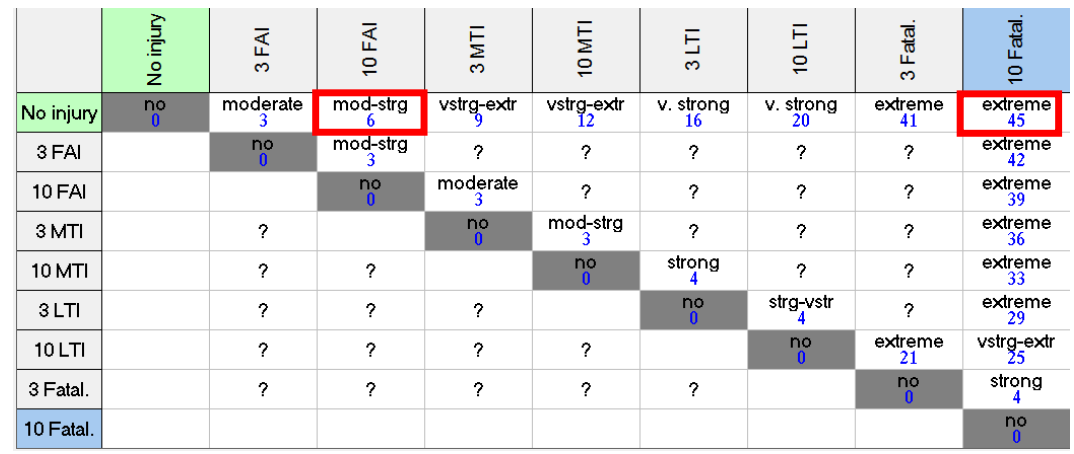

(a)

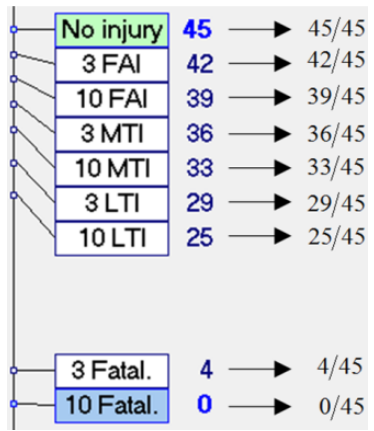

(b)

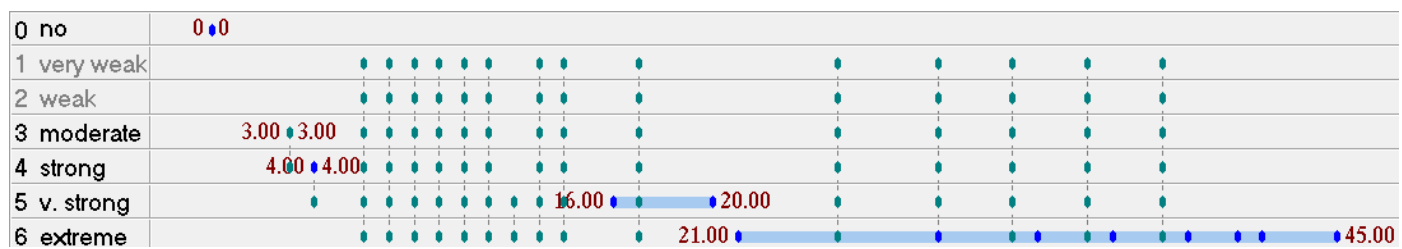

(c)

Figure 2: (a) Global MACBETH matrix of judgments, (b) numerical scales (left: basic MACBETH scale; right: MACBETH scale rescaled), (c) values or ranges of numerical values for the seven MACBETH semantic categories. 


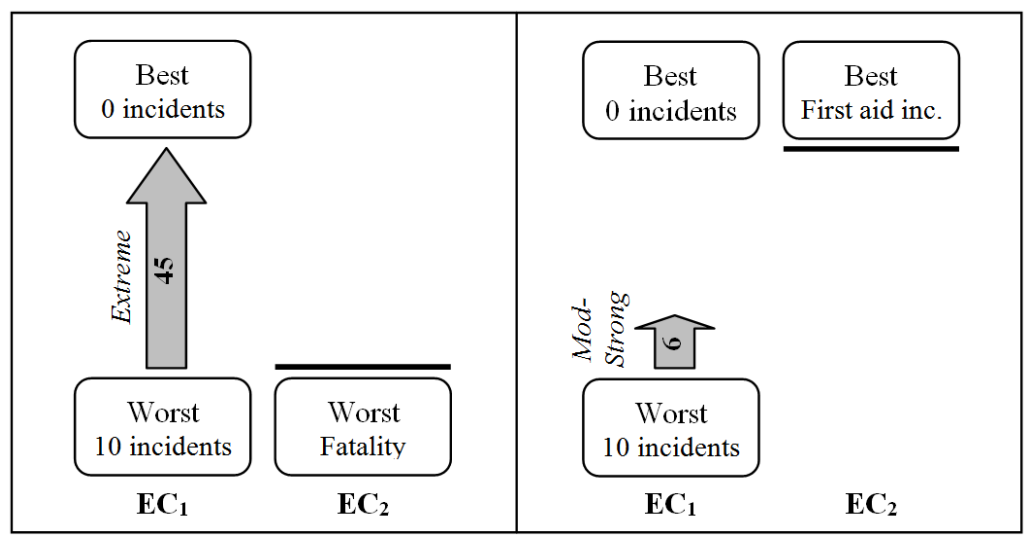

Figure 3: Illustrative image to help the understanding of Shapley parameter of the EC 1 "number of injuries".

and going from no fatality $\left(\mathrm{B}_{1} \mathrm{~W}_{2}\right)$ to no $\mathrm{FAI}\left(\mathrm{B}_{1} \mathrm{~B}_{2}\right)$. Mathematically, this parameter is given by:

$$
\left\{\begin{array}{l}
\mathrm{s}_{2}=\frac{1}{2} \times\left[\mathrm{V}_{\mathrm{Ag}}\left(\mathrm{W}_{1} \mathrm{~B}_{2}\right)-\mathrm{V}_{\mathrm{Ag}}\left(\mathrm{W}_{1} \mathrm{~W}_{2}\right)\right]+\frac{1}{2} \times\left[\mathrm{V}_{\mathrm{Ag}}\left(\mathrm{B}_{1} \mathrm{~B}_{2}\right)-\mathrm{V}_{\mathrm{Ag}}\left(\mathrm{B}_{1} \mathrm{~W}_{2}\right)\right] \\
=\frac{1}{2} \times\left(\frac{39}{45}-\frac{0}{45}\right)+\frac{1}{2} \times\left(\frac{45}{45}-\frac{45}{45}\right)=\frac{13}{30}
\end{array}\right.
$$

Regarding the interaction parameter, $\mathrm{I}_{\mathrm{A}}$, it can be interpreted as an average of the added value given by putting all the EC in a set A together (Grabisch, 1997), when all the baselines are considered. Mathematically, it is expressed by (7).

$$
\begin{aligned}
\mathrm{I}(\mathrm{A}) & =\sum_{\mathrm{K} \subset \mathrm{N} \backslash \mathrm{A}} \frac{(\mathrm{n}-|\mathrm{K}|-|\mathrm{A}|) !|\mathrm{K}| !}{(\mathrm{n}-|\mathrm{A}|+1) !} \sum_{\mathrm{L} \subset \mathrm{A}}(-1)^{|\mathrm{A}|-|\mathrm{L}|} \mathrm{V}_{\mathrm{Ag}}\left(\mathrm{B}_{\mathrm{K}} \cup \mathrm{B}_{\mathrm{L}}\right), \\
\mathrm{A} & \subset \mathrm{N}, \mathrm{A} \neq \varnothing
\end{aligned}
$$

In case of interdependencies, the combined effect of having two best levels is more or less than the sum of individual effects (Bana e Costa et al., 2005). Thus, there is a parameter to correct this situation, that is, an interaction parameter named $\mathrm{I}_{12}$ (in the domain $[-1,1]$ with positive values standing for a synergistic behaviour between EC $\mathrm{i}$ and $\mathrm{j}$, negative values for an antagonistic behaviour between EC i and $\mathrm{j}$, and null values for no interaction between $\mathrm{EC}$ $i$ and $\mathrm{j}$ ), as computed in eq. (8).

$$
\begin{aligned}
\mathrm{V}_{\mathrm{Ag}}(\mathrm{B} 1 \mathrm{~B} 2)-\mathrm{V}_{\mathrm{Ag}}(\mathrm{W} 1 \mathrm{~W} 2) & =\mathrm{V}_{\mathrm{Ag}}(\mathrm{B} 1 \mathrm{~W} 2)-\mathrm{V}_{\mathrm{Ag}}(\mathrm{W} 1 \mathrm{~W} 2)+ \\
& +\mathrm{V}_{\mathrm{Ag}}(\mathrm{W} 1 \mathrm{~B} 2)-\mathrm{V}_{\mathrm{Ag}}(\mathrm{W} 1 \mathrm{~W} 2) \\
& +\mathrm{I}_{12}
\end{aligned}
$$

Rewriting eq. (8) and using the rescaled value scores presented in Fig. 2(b), the $I_{12}$ parameter assumes the value of $-39 / 45$. This value is negative, being in line with expectations since there is an antagonism between the two EC. I.e., according to the DM, independently of the EC, a single best level has almost the same attractiveness as two best levels, since there is a very low difference in value between the referred options - see Fig. 2(b). Said in another way, no injury of any type of incident or any number of FAI has almost the same attractiveness as no FAI.

\section{CONCLUSIONS}

There is scope for developing tools to assist the modelling of interdependent EC for decision aid. This paper has identified some problems (such as the use of local judgments to model interdependencies) and challenges from using CI operators, proposing a simple procedure to use the CI operators with MACBETH. We have shown how a single MACBETH matrix can be populated with global (qualitative) judgments, by applying the MACBETH protocol of questioning and method, and how it can be coupled with CI operators. This approach allows for: (i) detecting inconsistent judgments and modelling cases of differences in opinion or hesitation; (ii) including more judgments than the ones required in some of the previous studies (promoting more robust results), if that is found appropriate in the decision context; and (iii) using the M-MACBETH DSS to assist the whole process with the DM (for instance, detecting inconsistencies and including judgments of hesitation or of differences in opinion). This study has illustrated how one single MACBETH matrix can be the starting point to test the compatibility of judgments with the 2-additive CI formulation; and attempted to provide a more intuitive explanation on the values of the Shapley and interaction parameters. 
Many aspects related to the use of a global MACBETH matrix require further research. First, the proposed approach needs to be applied to real cases (and we are doing so in the health and safety context). Second, it is relevant to analyse how the global matrix can assist in cases in which there are infeasible combinations between impact levels of different EC, such as with the EC of the reputation case developed in Bana e Costa et al. (1999). In fact, some combinations of impact levels across EC can be infeasible, leading for instance that levels such as $\left(\mathrm{W}_{1} \mathrm{~W}_{2} \mathrm{~B}_{3}\right),\left(\mathrm{W}_{1} \mathrm{~B}_{2} \mathrm{~W}_{3}\right)$ and $\left(\mathrm{W}_{1} \mathrm{~B}_{2} \mathrm{~B}_{3}\right)$ cannot be observed in the decision context, and therefore it is not possible to build "elementary expressions" using the same baseline. Third, it is worth exploring the compatibility of the global MACBETH matrix with the general formulation of the CI operators for more than two EC and with other mathematical formulations, such as with the multilinear one. Finally, it seems pertinent to exploit the CI mathematical formulation to model non-monotonic decision problems which involve not only cardinally but also ordinally interdependent EC.

\section{ACKNOWLEDGEMENTS}

This work was funded by National Funds from the Portuguese Public Budget through FCT - Fundação para a Ciência e a Tecnologia, within the project PTDC/EGE-GES/119230/2010.

\section{REFERENCES}

Aven, T., 2008. Risk analysis: assessing uncertainties beyond expected values and probabilities, Wiley.

Bana e Costa C. A., Ensslin L. and Costa A.P., 1998. Structuring the process of choosing rice varieties at the south of Brazil. Multi-Criteria Evaluation in Land-Use Management, Beinat E., Nijkamp P.(eds.), Kluwer Academic Publishers, Environment \& Management. 9: 33-45.

Bana e Costa, C.A. and Beinat, E., 2005. Modelstructuring in public decision-aiding. Working Paper LSE OR 05.79 - London School of Economics.

Bana e Costa, C.A., Carnero, M.C. and Oliveira, M.D., 2012. A multi-criteria model for auditing a Predictive Maintenance Programme. European Journal of Operational Research 217: 381-393.

Bana e Costa, C.A., Corrêa, E.C., de Corte, J.-M. and Vansnick, J.-C., 2002. Facilitating bid evaluation in public call for tenders: a socio-technical approach. The Internation Journal of Management Science 30: 227242.
Bana e Costa, C.A., de Corte, J.-M. and Vansnick, J.-C., 2012. MACBETH. International Journal of Information Technology \& Decision Making 11(2): 359-387.

Bana e Costa, C.A., Ensslin, L., Corrêa, E.C. and Vansnick, J.-C., 1999. Decision Support Systems in action: Integrated application in a multicriteria decision aid process. European Journal of Operational Research 113: 315-335.

Bana e Costa, C.A., Lourenço, J.C., Chagas, M.P. and Bana e Costa, J.C., 2008. Development of reusable bid evaluation models for the Portuguese Electric Transmission Company. Decision Analysis 5(1): 2242.

Barin, A., Canha, L.N., Abaide, A.R., Orling, R.B. and Martins, L.F.G., 2012. Selection of renewable energy technologies in landfills: The M-MACBETH decision support. Fuelling the Future: Advances in Science and Technologies for Energy Generation, Transmission and Storage. A. Mendez-Vilas, Universal-Publishers: 98-102.

Berrah, L. and Clivillé, V., 2007. Towards an aggregation performance measurement system model in a supply chain context. Computers in Industry 58: 709-719.

Bottero, M., Ferretti, V. and Mondini, G., 2013. A Choquet integral-based approach for assessing the sustainability of a new waste incinerator. International Journal of Multicriteria Decision Making 3(2/3): 157.

Brosig, J., 2012. Multi-criteria evaluation of classical swine fever control strategies using Choquet integral (chapter three). Alternative Classical Swine Fever control strategies - a simulation study. Agricultural and Food Sciences. Christian-Albrechts, University of Kiel. PhD thesis.

Cardin, M., Corazza, M., Funari, S. and Giove, S., 2013. Building a global performance indicator to evaluate academic activity using fuzzy measures. Neural Nets and Surroundings. Apolloni B., Bassis S., Esposito A. and Morabito F. C., Springer Berlin Heidelberg. 19: 217-225.

Carnero, M.C., 2006. Predictive maintenance programme audit with MACBETH. Safety and Reliability for Managing Risk. C. G. Soares and E. Zio, Taylor \& Francis Ltd. 1: 109-115.

Choquet, G., 1953. Theory of capacities. Annales de l'Institut Fourier 5: 131-295.

Clivillé, V., Berrah, L. and Mauris, G., 2007. Quantitative expression and aggregation of performance measurements based on the MACBETH multi-criteria method. International Journal of Production Economics 105: 171-189.

Clivillé, V., Mauris, G. and Berrah, L., 2006. A quantified industrial performance measurement system based on a Choquet fuzzy integral. IEEE International Conference on Fuzzy Systems, Vancouver, BC, Canada.

Edwards, W. and Barron, F.H., 1994. SMARTS and SMARTER: Improved simple methods for multiattribute utility measurement. Organizational Behavior and Human Decision Processes 60: 306-325. 
Feyziog̃lu, O. and Büyüközkan, G., 2010. Evaluation of Green Suppliers Considering Decision Criteria Dependencies. Multiple Criteria Decision Making for Sustainable Energy and Transportation Systems. Ehrgott M., Naujoks B, Stewart T.J. and W. J., Springer Berlin Heidelberg. 634: 145-154.

Figueiredo, M.S.M. and Oliveira, M.D., 2009. Prioritizing risks based on multicriteria decision aid methodology: Development of methods applied to ALSTOM power. IEEE International Conference on Industrial Engineering and Engineering Management, Hong Kong.

Grabisch, M., 1997. k-order additive discrete fuzzy measures and their representation. Fuzzy Sets and Systems 92: 167-189.

Grabisch, M., 2005. Une approach constructive de la décision multicritère. Traitment du Signal 22(4): 321 337.

Grabisch, M. and Labreuche, C., 2004. Fuzzy measures and integrals in MCDA. Multiple Criteria Decision Analysis: State of the Art Surveys. Figueira J., Greco S. and Ehrgott M., Kluwer Academic Publishers: 563608.

Grabisch, M. and Labreuche, C., 2010. A decade of application of the Choquet integral and Sugeno integrals in multicriteria decision-aid. Annals of Operations Reasearch 175(1): 247-290.

Grabisch M., Kojadinovic I. and Meyer P., 2008. A review of methods for capacity identification in Choquet integral based multi-attribute utility theory applications of the Kappalab R package. European Journal of Operational Research 186: 766-785.

Grabisch M. and Roubens M., 2000. Application of the Choquet integral in multicriteria decision making. Fuzzy Measures and Integrals - Theory and Applications. Grabisch M., Murofushi T. and Sugeno M., Physica Verlag: 348-374.

Gürbüz, T., 2010. Multiple criteria human performance evaluation using Choquet integral. International Journal of Computational Intelligence Systems 3(3): 290-300.

Keeney, R.L., 1992. Value-Focused Thinking: a path to creative decision making. Cambridge, Harvard University Press.

Labreuche, C. and Grabisch, M., 2003. The Choquet integral for the aggregation of interval scales in multicriteria decision making. Fuzzy Sets and Systems 137: 11-26.

Labreuche C. and Le Huéde F., 2005. Myriad: a tool suite for MCDA. International Conference of the Euro Society for Fuzzy Logic and Technology (EUSFLAT), Barcelona, Spain.

Linkov, I., Satterstrom, F.K., Kiker, G., Batchelor, C., Bridges, T. and E., F., 2006. From comparative risk assessment to multi-criteria decision analysis and adaptive management: Recent developments and applications. Environment International 32: 10721093.

Marichal, J.-L., 2000. An axiomatic approach of the discrete Choquet integral as a tool to aggregate interacting criteria. IEEE Transactions on Fuzzy Systems 8(6): 800-807.

Mayag, B., Grabisch, M. and Labreuche, C., 2011. A characterization of the 2-additive Choquet integral through cardinal information. Fuzzy Sets and Systems 184: 84-105.

Merad, M., Dechy, N., Serir, L., Grabisch, M. and Marcel, F., 2013. Using a multi-criteria decision aid methodology to implement sustainable development principles within an organization. European Journal of Operational Research 224: 603-613.

Morton, A. and Fasolo, B., 2009. Behavioural decision theory for multi-criteria decision analysis: a guided tour. Journal of the Operational Research Society $\mathbf{6 0}$ 268-275.

Shah, L.A., Etienne, A., Siadat, A. and Vernadat, F.B., 2013. Value-risk graph: a decision-making tool for supply chain and industrial system engineering. 6th IFAC International Conference on Management and Control of Production and Logistics. September 1113, Fortaleza, Ceará, Brazil.

Vernadat, F., Shah, L., Etienne, A. and Siadat, A., 2013. VR-PMS: a new approach for performance measurement and management of industrial systems. International Journal of Production Research: 1-19.

von Winterfeldt D. and Edwards W., 1986. Decision analysis and behavioral research. Cambridge, Cambridge University Press.

Williams, T.M., 1996. The two-dimensionality of project risk. International Journal of Project Management 14(3): 185-186. 